\title{
Non-digestible oligosaccharides scFOS/IcFOS facilitate safe subcutaneous immunotherapy for peanut allergy
}

Laura Wagenaar ${ }^{1}$,, Manon van Roest ${ }^{1}$, Laura J. W. Kruijssen ${ }^{1}$, Peter J. Simons ${ }^{4}$, Louis Boon4, Marlotte M. Vonk ${ }^{2,3}$, Betty C. A. M. van Esch ${ }^{2,3}$, Leon M. J. Knippels ${ }^{2,3}$, Johan Garssen ${ }^{2,3}$, Raymond H. H. Pieters ${ }^{1 *}$ and Joost J. Smit ${ }^{1}$

\begin{abstract}
Background: Improving the safety of subcutaneous immunotherapy (SCIT) for food allergy is necessary to reduce side effects and achieve long-term tolerance. We determined the effect of dietary supplementation with 1\% nondigestible short- and long-chain fructo-oligosaccharides (scFOS/ICFOS) on safety and efficacy of SCIT using a peanut allergy mouse model.

Methods: After sensitization, mice received a scFOS/ICFOS or control diet for the rest of the study. To study safety of SCIT, mice were dosed with a single subcutaneous injection of peanut extract (PE) or PBS. To study efficacy, mice were dosed subcutaneously (SCIT, 3 times/week) with PE or PBS for 3 weeks. Hereafter, acute allergic skin responses, anaphylactic shock symptoms and body temperature were assessed. To study the mechanism in vitro, the human IgE receptor (FceRI)-transfected rat mast cell (RBL) line was sensitized with an oligoclonal pool of chimeric human (chu)lgE antibodies against bovine $\beta$-lactoglobulin (BLG) and incubated with the oligosaccharides before exposure to BLG to assess direct the effect on degranulation.

Results: SCFOS/ICFOS reduced anaphylaxis caused by a single PE SCIT dose. sCFOS/lcFOS alone also reduced the acute allergic skin response. Moreover, scFOS/ICFOS supplementation resulted in lower MMCP-1 levels in serum after PE SCIT dose compared to control diet, while antibody levels were not affected by the diet. In vitro incubation with scFOS/IcFOS at $0.5 \%$ suppressed the degranulation of IgE-sensitized RBL cells. However, dietary supplementation with sCFOS/ICFOS did not improve the efficacy of SCIT.
\end{abstract}

Conclusions: We show that scFOS/ICFOS diet improves the safety of SCIT, as evidenced by lower anaphylactic responses without compromising the efficacy in a mouse model for peanut allergy. This effect is likely to result from the suppression of mast cell effector function.

Keywords: Peanut allergy, Immunotherapy, Mast cells, Non-digestible oligosaccharides

\section{Background}

Food allergy is a major public health issue in Western countries, as it affects $8 \%$ of American children, of which most of them are peanut allergic (25\%) [1]. Currently, food allergy can only be managed by strict avoidance of the causative food and in case of accidental exposure,

\footnotetext{
*Correspondence: r.h.h.pieters@uu.nl

1 Department of Immunotoxicology, Faculty of Veterinary Medicine, Institute for Risk Assessment Sciences, Utrecht University, Yalelaan 104 3508 TD Utrecht, The Netherlands

Full list of author information is available at the end of the article
}

with anaphylactic rescue medication. Therefore, a safe therapy leading to persistent tolerogenic protection is highly needed.

For many years, desensitization and/or tolerance induction to allergens via allergen-specific immunotherapy (AIT) has been the focus of research. AIT using the subcutaneous, oral, or sublingual route provided encouraging results in food allergy, despite serious and significant safety concerns [2-8]. Two small studies in peanut allergic patients showed that subcutaneous immunotherapy (SCIT) was associated with reduced symptoms 
$[9,10]$. However, a high rate of serious systemic reactions (13\%) made this treatment unsafe for routine use. Because of these limitations, although effective, it is currently not recommended to use immunotherapy for peanut allergy for routine clinical use [11-13].

Combining AIT with a nutritional intervention may provide a new window of opportunity to improve the efficacy and safety of AIT for food allergic patients. The combination of SCIT or sublingual immunotherapy for allergic rhinitis or asthma with bacterial products or Tolllike receptor ligands has demonstrated enhanced and persistent beneficial effects in both animals [14-16] and patients $[17,18]$. However, human data concerning the additive effect of supplementation with immunomodulatory food components on the safety and efficacy of OIT for food allergy is limited. In peanut allergic patients, combining OIT with a probiotic strain resulted in a long-lasting clinical benefit $[19,20]$. Besides probiotics, prebiotic components like dietary non-digestible oligosaccharides, derived from vegetable or dairy sources, also support growth of beneficial bacteria in the gut [21]. These oligosaccharides, like short- and long-chain fructooligosaccharides (scFOS/lcFOS), were able to effectively prevent the onset of allergy, and prevent allergic manifestations in different mouse models [22-24]. Moreover, in whey-sensitized mice a diet supplemented with scFOS/ lcFOS showed improved efficacy of OIT [25]. Therefore, non-digestible oligosaccharides administered after sensitization but before SCIT, might provide a better safety profile during treatment and therefore might improve the efficacy of therapy.

A food allergic reaction is induced by the fast, local and systemic release of inflammatory mediators such as histamine, serotonin, and various pro-inflammatory cytokines from mast cells and basophils [26]. Consequently, we hypothesized that scFOS/lcFOS could have an effect on the basophil effector function.

In the current study, it was investigated whether dietary supplementation with scFOS/lcFOS can maintain the effectiveness in the absence of side-effects of SCIT by reducing the allergic response seen after a single SCIT dose was administered. Moreover, it was discovered that these oligosaccharides have a direct inhibitory effect on degranulation of mast cells.

\section{Materials and methods}

Mice

Female $\mathrm{C} 3 \mathrm{H} / \mathrm{HeOuJ}$ mice (5-6-week-old) purchased from Charles River Laboratories (Erkrath, Germany) were maintained under controlled conditions (relative humidity of $50-55 \%, 12 \mathrm{~h}$ light/dark cycle, temperature of $23 \pm 2{ }^{\circ} \mathrm{C}$ ). The mice were housed at the animal facility of Utrecht University in filter-topped macrolon cages ( $\mathrm{n}=6-8$ per cage/group), with wood chipped bedding, tissues and a plastic shelter and food and water were provided ad libitum. An independent ethics committee for animal experimentation (the Ethical Committee of Animal Research of Utrecht University, Utrecht, The Netherlands) approved animal procedures. All procedures complied with the principles of good laboratory animal care following the European Directive for the protection of animals used for scientific purposes.

\section{Reagents and diets}

Peanut protein extract (PE, $30 \mathrm{mg} / \mathrm{ml}$ ) was prepared from raw peanuts (provided by Intersnack Nederland BV, The Netherlands) as described previously [27], checked for protein content by BCA analysis (Pierce, IL) and kept at $-20{ }^{\circ} \mathrm{C}$ until use. The adjuvant cholera toxin $(\mathrm{CT})$ was acquired from List Biological Laboratories Inc. (Campbell, CA, USA).

Ssniff Spezialdiäten (Soest, Germany) composed the semi-purified peanut protein-free AIN-93G-based diets. The scFOS/lcFOS diet was supplemented with nondigestible oligosaccharides, which consisted of a 9:1, 1\% (w/w) mixture of short-chain fructo-oligosaccharides (scFOS: oligofructose; Raftilose P95, Orafti, Wijchen, the Netherlands; $>95 \%$ degree of polymerization [DP] $<6)$ and long-chain fructo-oligosaccharides (lcFOS: long chain inulin; Raftiline HP, Orafti, Wijchen, the Netherlands; average DP 23 or higher, $<1 \% \mathrm{DP}<5$ ) derived from chicory inulin. The AIN-93G diet without scFOS/lcFOS supplementation was used as control diet. The diets were stored at $4{ }^{\circ} \mathrm{C}$ prior to use.

\section{Experimental designs}

Upon arrival, mice were randomly divided over the control and experimental groups and were fed control diet. To study the potential of scFOS/lcFOS to improve the safety and efficacy of SCIT, two study treatment protocols were used (Fig. 1). In both protocols, mice $(n=6-8)$, were sensitized to PE i.g. (6 mg PE, $200 \mu \mathrm{l} /$ mouse) with CT $(15 \mu \mathrm{g} /$ mouse $)$ on three consecutive days, followed by a weekly dosing for 4 weeks (PE), as previously described [27]. Sham-sensitized mice received CT in PBS alone.

The effect of scFOS/lcFOS on the safety of SCIT was studied in a peanut allergy mouse model (treatment protocol 1, Fig. 1). Depicted groups received the scFOS/ lcFOS supplemented diet from day 35 until the end of the study. On day 42, anaphylactic shock symptom scores, body temperature levels and mucosal mast cell degranulation after one high dose of SCIT (PE $100 \mu \mathrm{g}$ s.c. or PBS) were determined. At the end of the study (day 43), 


\section{Treatment protocol 1. Safety}

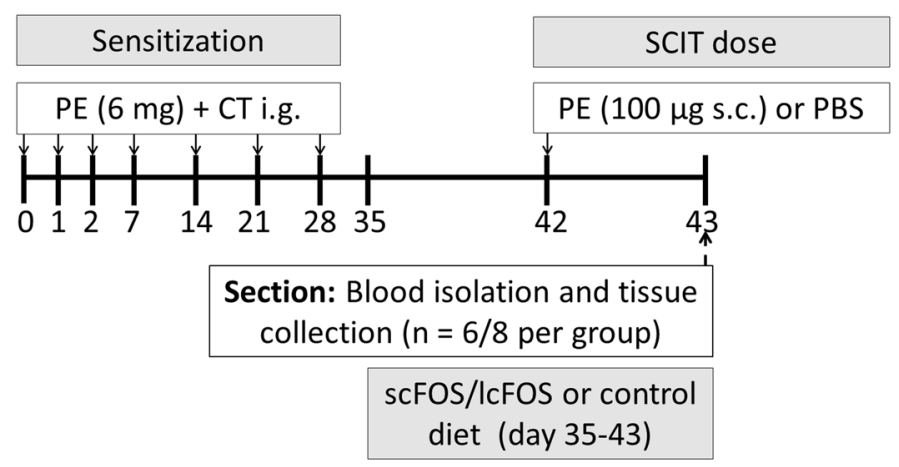

\section{Treatment protocol 2. Efficacy}

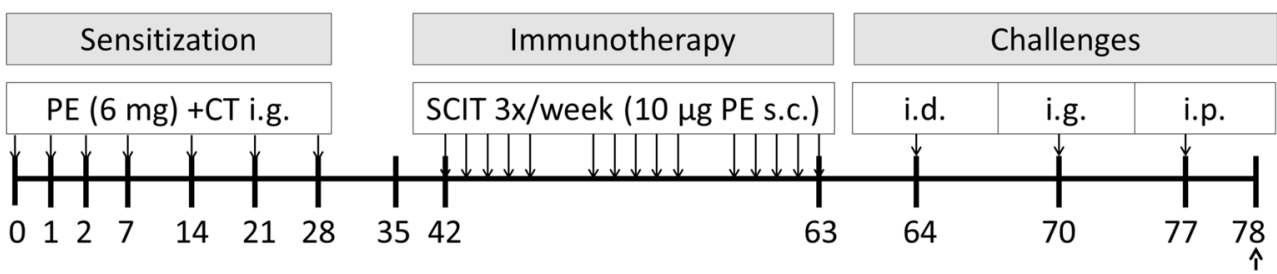

Section: Blood isolation and tissue collection

( $n=6 / 8$ per group)

scFOS/IcFOS or control diet (day 35-78)

Fig. 1 Schematic overviews of the experimental set-ups. Treatment protocol 1 Safety To study the ability of scFOS/IcFOS to improve the safety of SCIT in PE sensitized mice. Treatment protocol 2 Efficacy To study the ability of scFOS/lcFOS to improve the efficacy of SCIT in PE sensitized mice. PE, peanut extract; $C$ T, cholera toxin; SCIT, subcutaneous immunotherapy; s.c., subcutaneous; i.d., intradermal; i.g., intragastric; i.p., intraperitoneal

mice were killed by cervical dislocation and spleen was collected.

The effect of scFOS/lcFOS on the efficacy of SCIT was studied in the same peanut allergy mouse model (treatment protocol 2, Fig. 1). After sensitization, mice were treated for 3 weeks with PE, s.c. $(10 \mu \mathrm{g}$ PE/mouse $)$ three times a week. Sham-sensitized and PE-sensitized control mice were treated i.g. with PBS alone. The diet of selected groups was supplemented with scFOS/ lcFOS from day 28 (after sensitization) until the end of the study. On day 64, the acute allergic skin response was determined in all mice after i.d. exposure to PE in both ear pinnae with $1 \mu \mathrm{g}$ PE in $20 \mu \mathrm{l}$ PBS. On day 70 , MMCP-1 levels were determined in blood samples collected $30 \mathrm{~min}$ after mice were i.g. exposed to $15 \mathrm{mg}$ PE in $500 \mu \mathrm{l}$ PBS. To measure ear thickness, in duplicate prior to and $1 \mathrm{~h}$ after i.d. PE exposure in both ear pinnae, all mice were anesthetized using inhalation of isoflurane. To determine $\Delta$ ear swelling as a measure for the acute allergic skin response, basal ear thickness $(\mu \mathrm{m})$ was subtracted from the ear thickness $1 \mathrm{~h}$ post-challenge. Anaphylactic shock symptom scores and body temperature levels were determined after i.p. exposure on day 77 with $100 \mu \mathrm{g}$ PE in $200 \mu \mathrm{l}$ PBS. Body temperature was measured every $10 \mathrm{~min}$ after the i.p. or high SCIT and OIT challenge using a rectal thermometer and clinical symptoms were scored after $40 \mathrm{~min}$, according to the method described by Li et al. [28]. At the end of the experiment on day 78, the mice were killed by cervical dislocation and blood and organs were collected.

\section{Serum levels of MMCP-1 and allergen-specific $\lg E, \lg A$, IgG1 and IgG2a}

PE-specific IgA, IgE, IgG1 and IgG2a levels in serum were detected by ELISA as previously described [27]. Briefly, high-binding 96-wells plates (Costar 3590, Corning Incorporated, Corning, NY, USA) were coated at $4{ }^{\circ} \mathrm{C}$ with $10 \mu \mathrm{g} / \mathrm{ml} \mathrm{PE}$ in PBS (IgG1 and IgG2a) or with $1 \mu \mathrm{g} / \mathrm{ml}$ rat anti-mouse IgE or IgA (BD Biosciences, Alphen aan den Rijn, The Netherlands) in PBS ON. Plates were blocked with $0.5 \%$ BSA-ELISA buffer 
for $1 \mathrm{~h}$ (RT). Diluted serum samples were incubated for $2 \mathrm{~h}$ (RT). For detection, AP-coupled anti-IgG1 and IgG2a antibodies were incubated for $1 \mathrm{~h}$ (RT). Subsequently, $1 \mathrm{mg} / \mathrm{ml} \mathrm{p}$-nitrofenylphosphat in diethanolamine buffer was used for the color reaction, which was stopped with a $10 \%$ EDTA solution. To measure PEspecific IgE and IgA, PE-DIG conjugate solution (1 h RT) and peroxidase-conjugated anti-DIG fragments ( $1 \mathrm{~h}$ at $\mathrm{RT}$ in the dark) were added. After incubation, a tetramethylbenzidine substrate solution was used and the color reaction was stopped with $2 \mathrm{M} \mathrm{H}_{2} \mathrm{SO}_{4}$. Absorbance was measured at $405 \mathrm{~nm}$ (IgG1 and IgG2a) and at $450 \mathrm{~nm}$ (IgE and IgA) using an Asys expert 96 plate reader (Biochrom, Cambourne, UK). Concentrations of IgE, IgA, IgG1 and IgG2a were calculated in arbitrary units (AU) using a standard curve of pooled sera from PE-sensitized mice.

Serum obtained $30 \mathrm{~min}$ after i.g. challenge (treatment protocol 1 and 2, Fig. 1) was used to measure Mouse Mast Cell Protease-1 (MMCP-1). MMCP-1 was determined by using an MMCP-1 Sandwich ELISA kit (eBioscience MMCP-1 ELISA Ready-SET-Go Kit, Breda, The Netherlands) according to the manufacturer's instructions.

\section{Cytokine release after ex vivo stimulation of spleen lymphocytes with PE}

$8 \times 10^{5}$ cells derived from spleen were cultured $(200 \mu \mathrm{l} /$ well) in U-bottom culture plates (Greiner, Frickenhausen, Germany) using RPMI 1640 medium (Lonza, Verviers, Belgium) with $10 \% \mathrm{FCS}$, penicillin $(100 \mathrm{U} / \mathrm{ml}) /$ streptomycin $(100 \mu \mathrm{g} / \mathrm{ml})$ (Sigma). All cells were stimulated with culture medium as a negative control, a polyclonal stimulation with anti-CD3/CD28 $(1 \mu \mathrm{g} / \mathrm{ml}$, clone $145-2 \mathrm{C} 11$ and clone 37.51, eBioscience) or allergen-specific stimulation with PE $(100 \mu \mathrm{g} / \mathrm{ml})$. Interleukin (IL)-5, IL-10, IL-13 and Interferon- $\gamma$ (IFN- $\gamma$ ) production by $\mathrm{T}$ cells were determined after $48 \mathrm{~h}$ (anti-CD3/CD28) or $96 \mathrm{~h}$ (PE) incubation. Culture supernatants were collected and stored at $-20{ }^{\circ} \mathrm{C}$ until further analysis with the Ready-SET-Go! ${ }^{\circledR}$
ELISA (eBioscience) according to the manufacturer's instructions.

\section{RBL cell degranulation assay}

Human FceRI-expressing rat basophilic leukemia RBLSX38 cells transfected with a nuclear factor of activated T-cells (NFAT)-responsive luciferase reporter gene, were used to measure mast cell degranulation as previously described [29]. In short, these RBL cells were plated in clear bottom 96 well plates and sensitized using an oligoclonal pool of chimeric human (chu) IgE antibodies against bovine $\beta$-lactoglobulin (BLG, a major allergen in bovine whey), as described by Knipping et al. [30]. Hereafter, RBL cells were incubated for $24 \mathrm{~h}$ with 0.05 and $0.5 \%$ scFOS/lcFOS (ratio 9:1). To induce degranulation, cells were exposed to 1, 10, 100 and $1000 \mathrm{ng} / \mathrm{ml} \mathrm{BLG}$. After stimulation, luciferase substrate solution containing cell lysis reagent (One-Glo, Promega Corp., Tokyo, Japan) was added to the cells, and chemiluminescence was measured. Luciferase expression levels are represented as the fold increase of relative light units compared with the background expression, after subtraction of a blank control (without cells).

\section{Statistics}

For all statistical analyses, GraphPad Prism 6.0c software for Macintosh (GraphPad Software, San Diego, CA, USA) was used. Anaphylaxis symptom scores and cytokine levels were analyzed using Kruskal-Wallis test for nonparametric data with Dunn's post hoc test. Body temperature levels of the peanut allergy safety study were analyzed on each time-point by one-way ANOVA and Bonferroni's post hoc test to compare preselected groups. Body temperature levels for the efficacy study were analyzed using a one-way repeated measures ANOVA and Bonferroni's post hoc test. The acute allergic skin response was statistically analyzed by one-way ANOVA and Bonferroni's post hoc test for multiple comparisons to compare preselected groups. Serum MMCP-1 results were log-transformed and statistically analyzed by one-way ANOVA and Bonferroni's post hoc test for multiple comparisons

\footnotetext{
(See figure on next page.)

Fig. 2 Allergic manifestations evaluated in PE-sensitized mice after fed the scFOS/lcFOS supplemented diet and a singular SCIT dose. The effect of SCFOS/ICFOS on the safety of SCIT in a peanut allergy model, according to treatment protocol 1 (Fig. 1). a Change in body temperature after SCIT challenge on day 42. b Anaphylactic shock symptom scores determined 40 min after SCIT challenge on day 42 . c Concentrations of MMCP-1 in serum collected 30 min after SCIT challenge on day 42. d Cytokine concentrations (IL-5, IL-10, IL-13 and IFN- $\gamma$ ) after ex vivo stimulation of splenocytes with PE collected at day 43. e-h Allergen-specific lgE, IgA, lgG1 and IgG2a measured by ELISA in serum of mice. Data are represented as mean \pm SEM $n=6$ mice/group, experiments were performed twice independently. Statistical analysis was performed using a two-way repeated measures ANOVA with Bonferroni's post hoc test (body temperature), a one-way ANOVA with Bonferroni's post hoc test (MMCP-1 and antibody levels), or a Kruskal-Wallis test with Dunn's post hoc test (Clinical score and cytokine levels). For body temperature; \#\#\#P<0.0001; \#P<0.01 compared to the control group. ${ }^{* *} \mathrm{P}<0.01$ compared to same group on control diet. For other results; ${ }^{* * *} \mathrm{P}<0.001$; ${ }^{* *} \mathrm{P}<0.01 ;{ }^{*} \mathrm{P}<0.05 \mathrm{compared}$ to indicated group. S.C., subcutaneous; i.g. intra gastric; PE, peanut extract; CNTR, control group; FF, scFOS/ICFOS; MMCP-1, mucosal mast cell protease-1; IL-, interleukin; IT, immunotherapy
} 


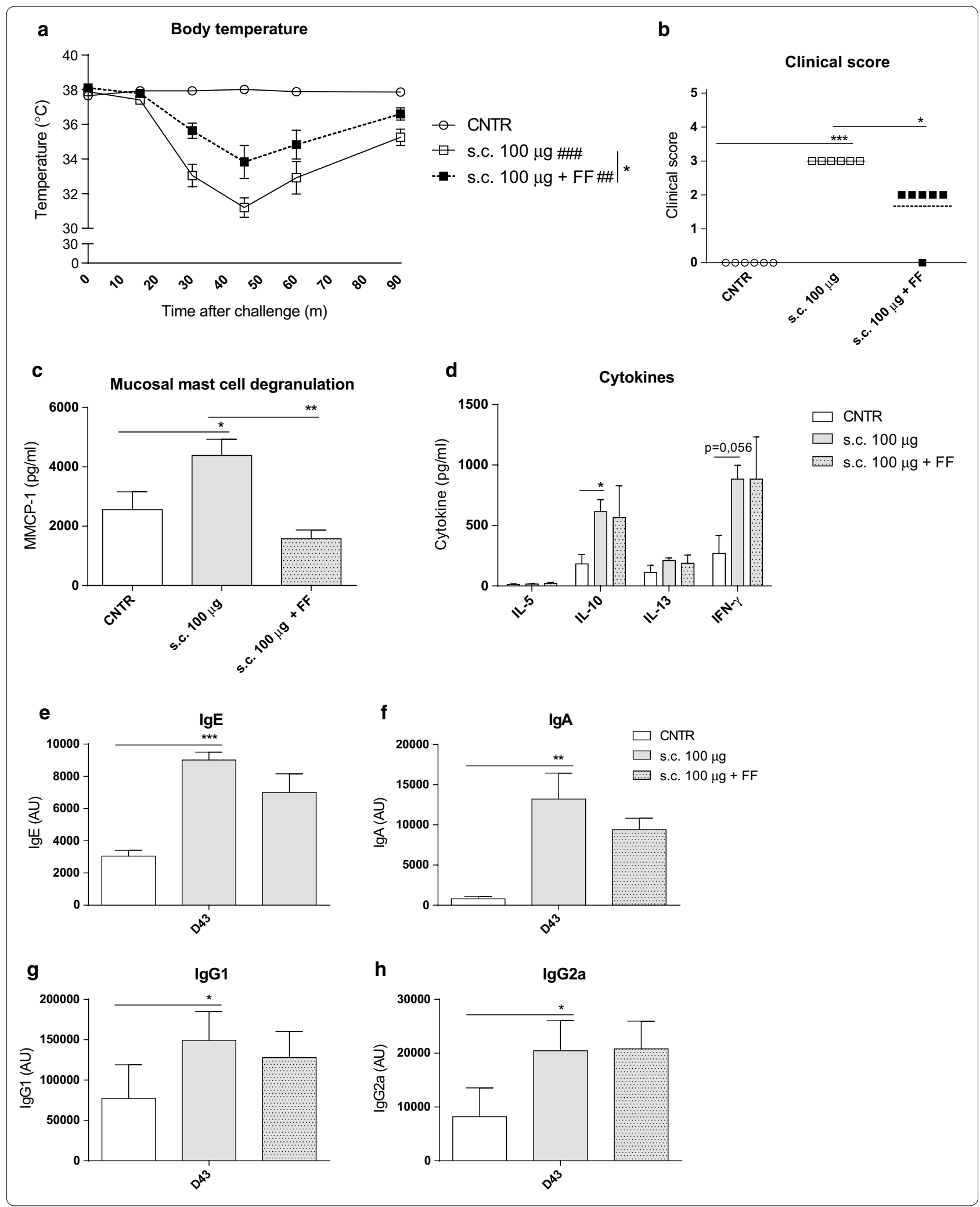




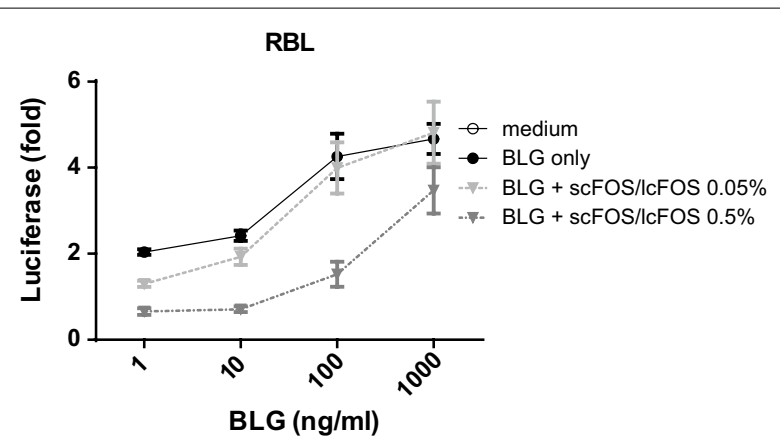

Fig. 3 RS-ALT8 cell degranulation. RS-ATL8 cells were sensitized with oligoclonal pool of chimeric human (chu)lgE antibodies and incubated with scFOS/LCFOS. IgE crosslinking-induced luciferase expression by 1, 10, 100 and $1000 \mathrm{mg} / \mathrm{ml}$ BLG or medium was shown. Data are represented as mean $\pm \operatorname{SEM}(n=2$ for 3 separate experiments)

to compare preselected groups. Immunoglobulin levels were depicted as mean $\pm S E M$, were log transformed prior to testing, and statistical difference compared to the PE-sensitized control treatment was analyzed each day by a one-way ANOVA and Dunnett's post hoc test. All data are presented as mean \pm SEM of 5-8 mice per group and results were considered statistically significant when $\mathrm{P}<0.05$.

\section{Results \\ scFOS/IcFOS reduced anaphylaxis and mast cell degranulation after SCIT PE dose}

The protective effect of scFOS/lcFOS supplementation to reduce side effects induced by SCIT was examined by analyzing allergic responses after one SCIT PE dose (Fig. 2). A single dose of $100 \mu$ g s.c. caused an anaphylactic response in PE-sensitized mice, measured by a severe drop in body temperature and high anaphylactic symptom scores compared to the mice receiving a PBS dose (Fig. 2a, b). Importantly, mice that were supplemented with scFOS/lcFOS after sensitization had a significant lower anaphylactic response (Fig. 2a, b).

In addition, exposure of PE-sensitized mice to a $100 \mu \mathrm{g}$ SCIT dose after supplementation with scFOS/lcFOS resulted in lower serum MMCP-1 levels compared to the group fed the control diet (Fig. 2c).

Cytokine production was measured in culture supernatant of PE-restimulated splenocytes obtained on day 43 (Fig. 2d). Splenocytes from SCIT-treated mice, both in the presence and absence of scFOS/lcFOS, showed an increased PE-induced IL-10 production when compared to control-treated mice (Fig. 2d). scFOS/lcFOS feeding did not change the cytokine production (Fig. $2 \mathrm{~d}$ ). In addition, scFOS/lcFOS feeding of PE-sensitized mice did not change antibody levels in serum compared to mice on control diet (Fig. 2e, f).

Noticeably, scFOS/lcFOS supplementation after sensitization considerably reduced side effects of SCIT possibly by an inhibitory effect on mast cell degranulation.

\section{Incubation with $\mathbf{0 . 5 \%}$ scFOS/IcFOS lowered RBL degranulation}

Since there was indication based on MMCP-1 levels that mast cells were involved in this reduction, we next focused on the effect of these oligosaccharides on degranulation of basophils in vitro. scFOS/lcFOS dosedependently inhibited the BLG-induced degranulation of anti-BLG IgE-sensitized RBL cells (Fig. 3).

\section{SCIT was able to effectively induce protection against anaphylaxis without improving effect of the dietary intervention}

The effect of scFOS/lcFOS on the efficacy of SCIT was examined by analyzing allergic responses after various PE exposures (Fig. 4). In PE-sensitized control mice, the i.p. challenge with $\mathrm{PE}$ elicited an anaphylactic response compared to the sham-sensitized control mice (Fig. 4a, b). This anaphylactic response was characterized by a sharp drop in body temperature and high clinical symptom scores. SCIT, with or without scFOS/lcFOS, resulted in a lower anaphylactic drop in body temperature compared to the PE-sensitized control mice, whereas scFOS/ lcFOS alone did not (Fig. 4a). Moreover, SCIT effectively decreased anaphylactic symptom scores compared to the non-treated PE-sensitized mice (Fig. 4b).

The i.d. PE-challenge resulted in an increased acute allergic skin response, as characterized by an ear swelling response $1 \mathrm{~h}$ after injection, in PE-sensitized control mice compared to sham-sensitized control mice (Fig. 4c). SCIT treatment did not change ear swelling compared to the PE-sensitized control mice (Fig. 4c). However, scFOS/lcFOS dietary supplementation resulted in a reduced ear swelling compared to the PE-sensitized control group again showing reduction of the allergic response (Fig. 4c).

Levels of MMCP-1, reflecting mucosal mast cell responses, were measured in serum collected $30 \mathrm{~min}$ after i.g. challenge (day 70, Fig. 4d). MMCP-1 levels were increased in PE-sensitized control mice compared to PBS-sensitized control mice (Fig. 4d). SCIT treatment in combination with scFOS/lcFOS resulted in lower MMCP-1 levels after i.g. challenge compared to only scFOS/lcFOS dietary intervention (Fig. 4d). 

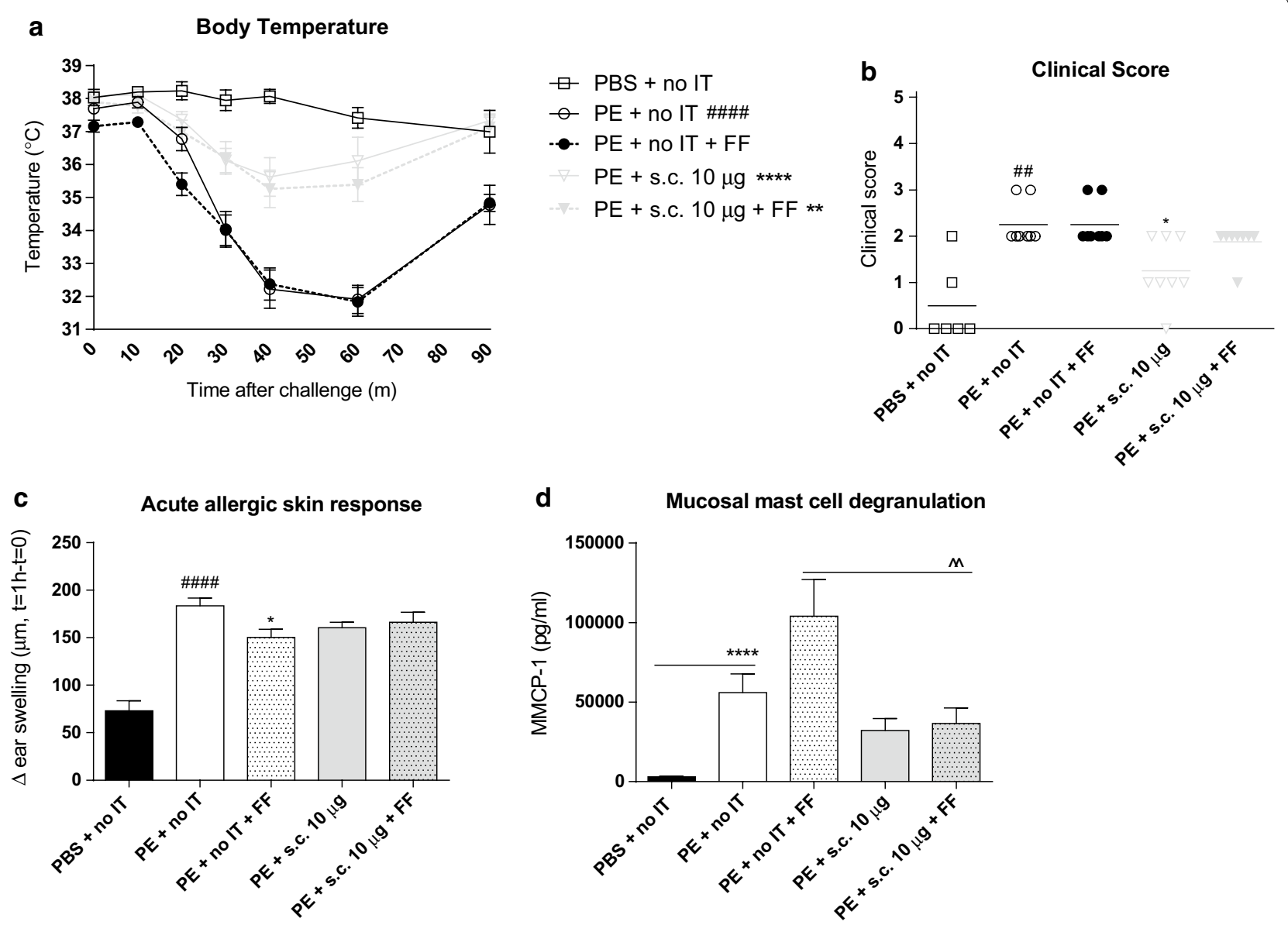

Fig. 4 Allergic manifestations evaluated in PE-sensitized mice after having received SCIT or SCIT in combination with scFOS/IcFOS. The effect of sCFOS/ICFOS on the efficacy of SCIT, according to treatment protocol 2 (Fig. 1). a Change in body temperature after intraperitoneal challenge on day 77. b Anaphylactic shock symptom scores determined $40 \mathrm{~min}$ after intraperitoneal challenge on day 77. c Acute allergic skin response measured as $\Delta$ ear swelling $1 \mathrm{~h}$ after intradermal challenge on day $64 . \Delta$ Ear swelling was calculated as average of left and right ear swelling subtracted with the basal ear thickness before challenge. $\mathbf{d}$ Concentrations of MMCP-1 in serum collected 30 min after intragastric challenge on day 70. Data are represented as mean \pm SEM $n=6-8$ mice/group. Statistical analysis was performed using a two-way repeated measures ANOVA with Bonferroni's post hoc test (body temperature) one-way ANOVA and Bonferroni's post hoc test (acute allergic skin response and MMCP-1) or a Kruskal-Wallis test with Dunn's post hoc test (Clinical score). ${ }^{\# \# \#} \mathrm{P}<0.0001$; ${ }^{\# \#} \mathrm{P}<0.01$ compared to sham-sensitized control. ${ }^{* * *} \mathrm{P}<0.0001$; ${ }^{* *} \mathrm{P}<0.01$; ${ }^{*} \mathrm{P}<0.05$ compared to PE-sensitized control. s.C., subcutaneous; $\mathrm{PE}$, peanut extract; FF, scFOS/ICFOS; MMCP-1, mucosal mast cell protease-1; IT, immunotherapy

\section{SCIT increased IgE and IgG levels}

On different time-points antibody concentrations were determined (Fig. 5). PE-sensitized mice showed enhanced IgE, IgG1 and IgG2a levels compared to the sham-sensitized mice (day 35, 50, 63, Fig. 5a-c). SCIT, with or without scFOS/lcFOS, first increased IgE and IgG1 and later IgG2a levels compared to non-treated PE-sensitized mice (day 50 and 63, Fig. 5a-c). The i.g. challenge induced IgG2a levels in SCIT-treated mice compared to nontreated PE-sensitized mice (day 70, Fig. 5c). After the i.p. challenge IgE levels increased in non-treated PE-sensitized mice but SCIT treatment protected against this increase (day 78, Fig. 5a).

\section{SCIT induced Th2 cytokine production in spleen}

To further study the effects of the treatments in reducing allergic symptoms, cytokine production by $\mathrm{T}$ cells was studied. No differences were found between cytokine concentrations upon allergen-specific stimulation of lymphocytes derived from PE-sensitized control mice and sham-sensitized control mice (Fig. 6a-d). scFOS/lcFOS supplementation did not affect the cytokine production (Fig. 6a-d). SCIT treatment, with or without the scFOS/ lcFOS, increased IL-5, IL-10 and IL-13 production by PEstimulated splenic lymphocytes when compared to PEsensitized control mice (Fig. 6a-c), showing that scFOS/ 


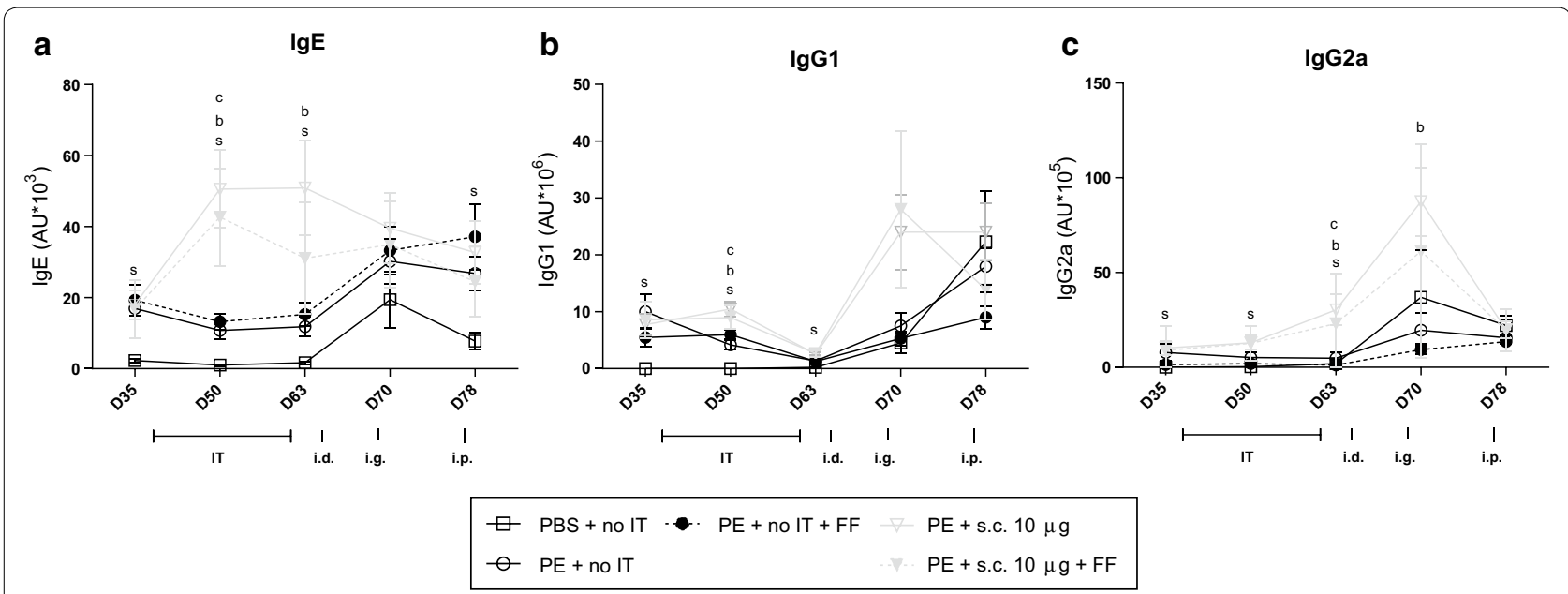

Fig. 5 PE specific antibody levels in serum. a-c Allergen-specific lgE, IgG1 and lgG2a measured by ELISA in serum of mice from treatment protocol 2 (Fig. 1). Data are represented as mean \pm SEM $n=6-8$ mice/group. Statistical analysis was performed, after log transformation, using a one-way ANOVA and Bonferroni's post hoc test (on each day). All treatment groups were compared to the PE-sensitized control group and significant differences were indicated with letters: a $\mathrm{P}<0.05$; aa $\mathrm{P}<0.01$; aaa $\mathrm{P}<0.001$; aaaa $\mathrm{P}<0.0001$. Letters used: $\mathrm{s}$ for $\mathrm{PBS}$-sensitized control group; a for scFOS/IcFOS control group; b for SCIT; c for SCIT plus scFOS/ICFOS. s.C., subcutaneous; i.g. intra gastric; i.p., intraperitoneal challenge; $\mathrm{PE}$, peanut extract; FF, scFOS/lcFOS; IT, immunotherapy

lcFOS did not impact the immunological modulation induced by SCIT.

\section{Discussion}

To reduce side-effects while maintaining efficacy of AIT for food allergy, several new treatment concepts have been investigated, as reviewed in [31-34]. The combination of AIT with nutritional interventions may provide a new possibility to enhance the safety and efficacy of AIT due to a reduction in side-effects during dose increasing protocols. Our results indicate that supplementation of the diet with scFOS/lcFOS reduced the side effects caused by a single PE SCIT dose. This effect appears to be due to a direct effect on mast cells, since scFOS/ lcFOS reduced mucosal mast cell degranulation in vivo and inhibited degranulation of RBL cells in vitro. These results support the concept that nutritional interventions have the potential to improve safety of SCIT.

The immunologic mechanisms underlying AIT are not fully understood and the observed desensitization or tolerance induction by AIT can occur via various, interrelated pathways [35]. Evidence from mouse models have shown that tolerance induction by OIT may be due to the activation of Tregs, i.e. CD4+CD25+FoxP3+ cells and IL-10- and TGF- $\beta$-producing Tregs $[25,36]$. This is reflected in OIT treated peanut allergic patients by significant changes in antigen induced $\mathrm{T}$-cell function and demethylation FoxP3 CpG sites after OIT [37]. In the used mouse model, OIT induced allergen-specific IgA and IgG1 antibodies [25, 38, 39]. Recently, IgG4 and IgA antibodies have been shown to be prominent immunoglobulins in the sustained regulation of food allergies in human immunotherapy trials $[6,40]$. In a murine model, mast cell degranulation and IgE-mediated systemic anaphylaxis induced by allergen ingestion were suppressed by allergen-specific IgG antibodies in the serum [41]. Accordingly, in a human/mouse chimeric model of respiratory allergy, it was demonstrated that post-AIT sera containing AIT-induced blocking antibodies is able to ameliorate allergic airway responses [42]. This suppressive effect of IgG might explain the ability of SCIT to reduce anaphylaxis after i.p. challenge.

This efficacy of SCIT to lower allergic manifestations, as shown by the decreased anaphylaxis after i.p. challenge, was not affected by scFOS/lcFOS. Peanut allergy in humans is Th2-dependent [43], during sensitization to peanut, priming of allergen-specific Th2 cells results in the production of Th2 cytokines (such as IL-4 and IL-13), which are responsible for class switching by B cells, allowing IgE production. In the current model, SCIT treatment showed a clear induction of cytokine production of IL-5, IL-13 and IL-10 by spleen-derived lymphocytes. These data may suggest that SCIT does not depend on reducing the $T h 2$ response, but rather on inducing the regulatory $\mathrm{T}$ cell response, which remained unaffected by scFOS/lcFOS.

Using a food allergy mouse model, it was shown that oral treatment with probiotics is able to reduce both systemic and local anaphylactic symptoms induced by oral challenge with the sensitizing allergen shrimp 

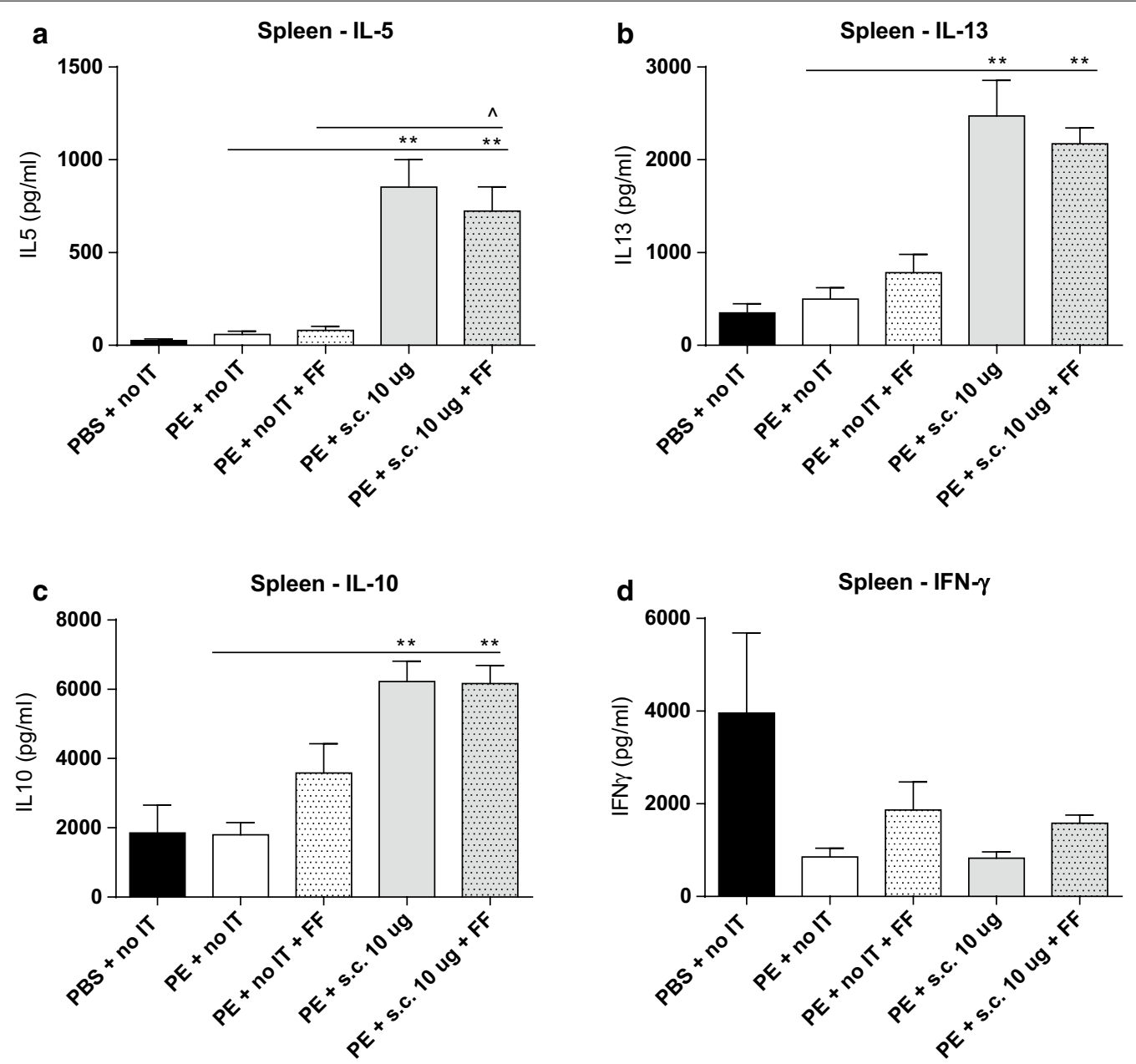

Fig. 6 Cytokine concentrations after ex vivo stimulation of spleen-derived lymphocytes with PE, determined by ELISA. Spleen-derived lymphocytes from day 78 were cultured for $96 \mathrm{~h}$ in the presence of PE or medium (treatment protocol 2, Fig. 1, medium data not shown). a IL-5 concentration. $\mathbf{b}$ IL-13 concentration. $\mathbf{c ~ I L - 1 0 ~ c o n c e n t r a t i o n . ~} \mathbf{d}$ IFN- $\gamma$ concentration. Data are represented as mean \pm SEM $n=6-8$ mice/group. Statistical analysis was performed using Kruskal-Wallis test with Dunn's post hoc test. ${ }^{* *} \mathrm{P}<0.01$ compared to PE-sensitized control. ${ }^{\wedge} \mathrm{P}<0.05$ compared to scFOS/IcFOS control. IT, immunotherapy; s.c., subcutaneous; PE, peanut extract; IL-, interleukin; FF, scFOS/IcFOS

tropomyosin [44]. Similar effects have been demonstrated in clinical trials, showing that probiotics are able to modulate the mucosal immune response, and that specific strains, especially lactic acid bacteria, are able to reduce allergic symptoms $[45,46]$. Besides probiotics, also prebiotic oligosaccharides are able to reduces the incidence of atopic dermatitis [47, 48]. However, studies using prebiotics for food allergy are limited, and the mechanism of clinical benefit is still unknown. Importantly, non-digestible oligosaccharides are detected in serum and urine of piglets fed galacto-oligosaccharides, meaning they can cross the gut epithelial barrier and could directly affect immune cells [49]. We show that $0.5 \%$ scFOS/lcFOS inhibit degranulation upon allergen challenge in vivo and in vitro. These findings are in line with those of $\mathrm{Xu}$ et al. who show that treatment with sulfated oligosaccharides, extracted from Eucheuma (E.) cottonii, lowered the serum levels of MMCP-1 after challenge in tropomyosin-allergic mice [50]. Moreover, these oligosaccharides were able to inhibit the secretion of allergy-related mediators like $\beta$-hexosaminidase, histamine and IL-4 and TNF- $\alpha$ [50].

We and others hypothesized that the combination of AIT with immunomodulatory components, which addresses both specific and unspecific (e.g. linked to innate effector cells such as mast cells) modulation of the immune response respectively, could be a promising strategy to improve efficacy and safety of AIT. Recently, we have shown that oligosaccharides are capable of improving the efficacy of OIT [39], and here we show that these substances also improve safety of SCIT. Previously it was shown that combining subcutaneous or 
sublingual immunotherapy with bacterial adjuvants or Toll-like receptor ligands could enhance tolerogenicity in allergic rhinitis $[14-16,51,52]$, a strategy which is already successfully translated to humans $[18,53-55]$. In peanut allergic children, OIT administration was combined with the probiotic Lactobacillus rhamnosus [19]. The authors report a long-lasting clinical benefit and persistent sustained unresponsiveness to peanut after 4 years without treatment [20]. Although no control (only OIT) was included, the proportion of children experiencing adverse events was lower compared to other trials where only OIT was used $[19,56,57]$. This study suggests that the combination of OIT and probiotics may lower the incidence of adverse events making this treatment clinically feasible.

\section{Conclusions}

In summary, we show that scFOS/lcFOS reduced anaphylaxis caused by a single PE SCIT dose, hereby improving the safety profile of SCIT in a mouse model. However, scFOS/lcFOS was not able to further improve the efficacy of SCIT in the current protocol. Nevertheless, when sideeffects are reduced higher dose of SCIT can be used for tolerance induction. Translated to clinical practice, the improvement of the safety profile could facilitate SCIT for peanut allergic patients more appropriate, although further studies are needed to determine the long-term supportive role of scFOS/lcFOS for AIT.

\begin{abstract}
Abbreviations
AIT: allergen-specific immunotherapy; PE: peanut extract; OIT: oral immunotherapy; SCIT: subcutaneous immunotherapy; i.g.: intragastric; CT: cholera toxin; i.d.: intradermal; i.p.: intraperitoneal; lg: immunoglobulin; MMCP-1: murine mast cell protease-1; IT: immunotherapy; Foxp3: forkhead box protein 3; Th: T helper; IL: interleukin; DP: degree of polymerization; IFNץ: interferon $\gamma$.
\end{abstract}

\section{Authors' contributions}

LW performed the literature search, wrote the animal applications, created the figures and wrote the manuscript. LW, MVR and LJWK performed data collection, analysis and interpretation. LW, RHHP and JJS designed the experimental set-up. JJS, RHHP, LB, PJS, MMV, BCAMVE, LMJK, and JG contributed to analysis and interpretation of data and manuscript writing. All authors read and approved the final manuscript.

\section{Author details}

${ }^{1}$ Department of Immunotoxicology, Faculty of Veterinary Medicine, Institute for Risk Assessment Sciences, Utrecht University, Yalelaan 104, 3508 TD Utrecht, The Netherlands. ${ }^{2}$ Department of Pharmacology, Faculty of Science, Utrecht Institute for Pharmaceutical Sciences, Utrecht University, Utrecht, The Netherlands. ${ }^{3}$ Department of Immunology, Danone Nutricia Research, Utrecht, The Netherlands. ${ }^{4}$ Bioceros BV, Utrecht, The Netherlands.

\section{Acknowledgements}

The authors would like to thank Marjolein Oosterveen-van der Doelen, for her technical assistance and Marianne Bol-Schoenmakers for the useful discussions. We also thank R. Nakamura (National Institute of Health Sciences, Tokyo, Japan) and F. H. Falcone (University of Nottingham, Nottingham, United Kingdom) for use of RBL-SX38 RBL reporter system.
Authors are part of the NUTRALL research consortium — Laura Wagenaar, Marlotte M. Vonk, Betty C. A. M. van Esch, Leon M. J. Knippels, Johan Garssen, Raymond H. H. Pieters, Joost J. Smit.

\section{Competing interests}

The authors declare that they have no competing interests; LK is employed by Nutricia Research and BE and JG are partly employed by Nutricia Research, Utrecht, The Netherlands.

\section{Availability of data and materials}

The datasets used and/or analyzed during the current studies are available from the corresponding author on reasonable request.

\section{Consent for publication}

Not applicable.

\section{Ethics approval and consent to participate}

All experimental procedures were approved by the Ethical Committee of Animal Research of Utrecht University and complied with the principles of good laboratory animal care following the European Directive for the protection of animals used for scientific purposes (registered by DEC2014.III.03.032 and AVD108002015212).

\section{Funding}

This research was financially supported by the STW 'Open Technology Program' grant and embedded in the NUTRALL consortium project entitled: "Nutrition-based approach to support antigen-specific immunotherapy for food allergies" (Grant Number 12652).

\section{Publisher's Note}

Springer Nature remains neutral with regard to jurisdictional claims in published maps and institutional affiliations.

Received: 30 May 2018 Accepted: 26 March 2019

Published online: 04 April 2019

\section{References}

1. Smallwood J, Dolen WK. The prevalence, severity, and distribution of childhood food allergy in the United States. Pediatrics. 2012;130:S10-1.

2. De Silva D, Geromi M, Panesar SS, Muraro A, Werfel T, Hoffmann-Sommergruber $\mathrm{K}$, et al. Acute and long-term management of food allergy: systematic review. Allergy Eur J Allergy Clin Immunol. 2014;69:159-67.

3. Vickery BP, Lin J, Kulis M, Fu Z, Steele PH, Jones SM, et al. Peanut oral immunotherapy modifies lgE and lgG4 responses to major peanut allergens. J Allergy Clin Immunol. 2013;131:128-134.e1-3. https://doi. org/10.1016/j.jaci.2012.10.048

4. Anagnostou K, Islam S, King Y, Foley L, Pasea L, Bond S, et al. Assessing the efficacy of oral immunotherapy for the desensitisation of peanut allergy in children (STOP II): a phase 2 randomised controlled trial. Lancet. 2014:383:1297-304.

5. Fleischer DM, Sicherer S, Greenhawt M, Campbell D, Chan E, Muraro A, et al. Consensus communication on early peanut introduction and the prevention of peanut allergy in high-risk infants. J Allergy Clin Immunol. 2015;136:258-61.

6. Burks AW, Jones SM, Wood RA, Fleischer DM, Sicherer SH, Lindblad RW, et al. Oral immunotherapy for treatment of egg allergy in children. N Engl J Med. 2012;367:233-43.

7. Skripak JM, Nash SD, Rowley H, Brereton NH, Oh S, Hamilton RG, et al. A randomized, double-blind, placebo-controlled study of milk oral immunotherapy for cow's milk allergy. J Allergy Clin Immunol. 2008;122:1154-60.

8. Elizur A, Appel MY, Goldberg MR, Yichie T, Levy MB, Nachshon L, et al. Clinical and laboratory 2-year outcome of oral immunotherapy in patients with cow's milk allergy. Allergy. 2016;71:275-8.

9. Oppenheimer JJ, Nelson HS, Bock SA, Christensen F, Leung DYM. Treatment of peanut allergy with rush immunotherapy. J Allergy Clin Immunol. 1992;90:256-62. 
10. Nelson HS, Lahr J, Rule R, Bock A, Leung D. Treatment of anaphylactic sensitivity to peanuts by immunotherapy with injections of aqueous peanut extract. J Allergy Clin Immunol. 1997;99:744-51.

11. Anagnostou K, Clark A. Oral immunotherapy for peanut allergy. Annu Rev Med. 2016;67:375-85.

12. Virkud YV, Burks AW, Steele PH, Edwards LJ, Berglund JP, Jones SM, et al. Novel baseline predictors of adverse events during oral immunotherapy in children with peanut allergy. J Allergy Clin Immunol. 2017;139(882-888):e5.

13. Vazquez-Ortiz M, Turner PJ. Improving the safety of oral immunotherapy for food allergy. Pediatr Allergy Immunol. 2015;27:117-25.

14. Lombardi V, Van Overtvelt L, Horiot S, Moussu H, Chabre H, Louise A, et al. Toll-like receptor 2 agonist Pam3CSK4 enhances the induction of antigen-specific tolerance via the sublingual route. Clin Exp Allergy. 2008:38:1819-29.

15. Van Overtvelt L, Moussu H, Horiot S, Samson S, Lombardi V, Mascarell L, et al. Lactic acid bacteria as adjuvants for sublingual allergy vaccines. Vaccine. 2010. https://doi.org/10.1016/j.vaccine.2010.02.009.

16. Van Overtvelt L, Lombardi V, Razafindratsita A, Saint-Lu N, Horiot S, Moussu $\mathrm{H}$, et al. IL-10-inducing adjuvants enhance sublingual immunotherapy efficacy in a murine asthma model. Int Arch Allergy Immunol. 2008. https://doi.org/10.1159/000108140.

17. Pfaar O, Barth C, Jaschke C, Hörmann K, Klimek L. Sublingual allergenspecific immunotherapy adjuvanted with monophosphoryl lipid A: a phase I/Ila study. Int Arch Allergy Immunol. 2011;154:336-44.

18. Drachenberg KJ, Wheeler AW, Stuebner P, Horak F. A well-tolerated grass pollen-specific allergy vaccine containing a novel adjuvant, monophosphoryl lipid A, reduces allergic symptoms after only four preseasonal injections. Allergy. 2001;56:498-505.

19. Tang MLK, Ponsonby AL, Orsini F, Tey D, Robinson M, Su EL, et al. Administration of a probiotic with peanut oral immunotherapy: a randomized trial. J Allergy Clin Immunol. 2015;135(737-744):e8.

20. Hsiao KC, Ponsonby AL, Axelrad C, Pitkin S, Tang MLK. Long term effects of a probiotic and peanut oral immunotherapy (PPOIT) treatment on peanut allergic children. J Allergy Clin Immunol. 2017;139:AB136.

21. Boehm G, Lidestri M, Casetta P, Jelinek J, Negretti F, Stahl B, et al. Supplementation of a bovine milk formula with an oligosaccharide mixture increases counts of faecal bifidobacteria in preterm infants. Arch Dis Child Fetal Neonatal Ed. 2002. https://doi.org/10.1136/fn.86.3.f178.

22. Kerperien J, Jeurink PV, Wehkamp T, van der Veer A, van de Kant HJG, Hofman GA, et al. Non-digestible oligosaccharides modulate intestinal immune activation and suppress cow's milk allergic symptoms. Pediatr Allergy Immunol. 2014;25:747-54.

23. Schouten B, van Esch BCAM, Hofman GA, Boon L, Knippels LMJ, Willemsen LEM, et al. Oligosaccharide-induced whey-specific CD25(+) regulatory T-cells are involved in the suppression of cow milk allergy in mice. J Nutr. 2010;140:835-41.

24. Vos AP, van Esch BC, Stahl B, M'Rabet L, Folkerts G, Nijkamp FP, et al. Dietary supplementation with specific oligosaccharide mixtures decreases parameters of allergic asthma in mice. Int Immunopharmacol. 2007;7:1582-7.

25. Vonk MM, Diks MAP, Wagenaar L, Smit JJ, Pieters RHH, Garssen J, et al. Improved efficacy of oral immunotherapy using non-digestible oligosaccharides in a murine cow's milk allergy model: a potential role for Foxp3+ regulatory T cells. Front Immunol. 2017;8:1230.

26. Kemp SF, Lockey RF. Anaphylaxis: a review of causes and mechanisms. J Allergy Clin Immunol. 2002;110:341-8.

27. van Wijk F, Hoeks S, Nierkens S, Koppelman SJ, van Kooten P, Boon L, et al. CTLA-4 signaling regulates the intensity of hypersensitivity responses to food antigens, but is not decisive in the induction of sensitization. J Immunol. 2005;174:174-9.

28. Li X, Schofield BH, Huang C-K, Kleiner Gl, Sampson HA. A murine model of IgE-mediated cow's milk hypersensitivity. J Allergy Clin Immunol. 1999;103:206-14.

29. Nakamura R, Uchida Y, Higuchi M, Nakamura R, Tsuge I, Urisu A, et al. A convenient and sensitive allergy test: IgE crosslinking-induced luciferase expression in cultured mast cells. Allergy Eur J Allergy Clin Immunol. 2010;65:1266-73.

30. Knipping K, Simons PJ, Buelens-Sleumer LS, Cox L, Den Hartog M, De Jong $N$, et al. Development of $\beta$-lactoglobulin-specific chimeric human IgEk monoclonal antibodies for in vitro safety assessment of whey hydrolysates. PLoS ONE. 2014;9:e106025. https://doi.org/10.1371/journ al.pone.0106025.

31. Leonard SA, Sampson HA, Sicherer SH, Noone S, Moshier EL, Godbold J, et al. Dietary baked egg accelerates resolution of egg allergy in children J Allergy Clin Immunol. 2012;130:473-480.e1. https://doi.org/10.1016/j. jaci.2012.06.006.

32. Leonard SA. Debates in allergy medicine: baked milk and egg ingestion accelerates resolution of milk and egg allergy. World Allergy Organ J. 2016. https://doi.org/10.1186/s40413-015-0089-5.

33. Gupta K, Kumar S, Das M, Dwivedi PD. Peptide based immunotherapy: a pivotal tool for allergy treatment. Int Immunopharmacol. 2014;19:391-8.

34. Smit J, Pieters R, van Roest M, Kruijssen L, Koppelman S, Opstelten D-J, et al. A novel peanut allergoid is safe and effective in immunotherapy in a peanut allergy mouse model. Clin Transl Allergy. 2013;3:P1.

35. Jones SM, Burks AW, Dupont C. State of the art on food allergen immunotherapy: oral, sublingual, and epicutaneous. J Allergy Clin Immunol. 2014;133:318-23.

36. Smaldini PL, Delgado MLO, Fossati CA, Docena GH. Orally-induced intestinal CD4+CD25+FoxP3+ treg controlled undesired responses towards oral antigens and effectively dampened food allergic reactions. PLoS ONE. 2015;10:e0141116. https://doi.org/10.1371/journal.pone.0141116.

37. Syed A, Garcia MA, Lyu SC, Bucayu R, Kohli A, Ishida S, et al. Peanut oral immunotherapy results in increased antigen-induced regulatory T-cell function and hypomethylation of forkhead box protein 3 (FOXP3). J Allergy Clin Immunol. 2014;133:500-10. https://doi.org/10.1016/j. jaci.2013.12.1037.

38. Vonk MM, Wagenaar L, Pieters RHH, Knippels LMJ, Willemsen LEM, Smit JJ, et al. The efficacy of oral and subcutaneous antigen-specific immunotherapy in murine cow's milk- and peanut allergy models. Clin Transl Allergy. 2017;7:35

39. Wagenaar L, Bol-Schoenmakers M, Giustarini G, Vonk MM, van Esch BCAM, Knippels LMJ, et al. Dietary supplementation with non-digestible oligosaccharides reduces allergic symptoms and supports low dose oral immunotherapy in a peanut allergy mouse model. Mol Nutr Food Res. 2018. https://doi.org/10.1002/mnfr.201800369.

40. Wright BL, Kulis M, Orgel KA, Burks AW, Dawson P, Henning AK, et al. Component-resolved analysis of lgA, lgE, and lgG4 during egg OIT identifies markers associated with sustained unresponsiveness. Allergy. 2016;71:1552-60.

41. Strait RT, Mahler A, Hogan S, Khodoun M, Shibuya A, Finkelman FD. Ingested allergens must be absorbed systemically to induce systemic anaphylaxis. J Allergy Clin Immunol. 2011;127(982-989):e1.

42. Vizzardelli C, Gindl M, Roos S, Möbs C, Nagl B, Zimmann F, et al. Blocking antibodies induced by allergen-specific immunotherapy ameliorate allergic airway disease in a human/mouse chimeric model. Allergy Eur J Allergy Clin Immunol. 2018. https://doi.org/10.1111/all.13363.

43. Turcanu V, Maleki SJ, Lack G. Characterization of lymphocyte responses to peanuts in normal children, peanut-allergic children, and allergic children who acquired tolerance to peanuts. J Clin Invest. 2003;111:1065-72.

44. Di Felice G, Barletta B, Butteroni C, Corinti S, Tinghino R, Colombo P, et al. Use of probiotic bacteria for prevention and therapy of allergic diseases: studies in mouse model of allergic sensitization. J Clin Gastroenterol. 2008. https://doi.org/10.1097/mcg.0b013e318169c463.

45. Kalliomäki M, Salminen S, Arvilommi H, Kero P, Koskinen P, Isolauri E. Probiotics in primary prevention of atopic disease: a randomised placebo-controlled trial. Lancet. 2001. https://doi.org/10.1016/s0140 $-6736(00) 04259-8$.

46. Ozdemir O. Various effects of different probiotic strains in allergic disorders: an update from laboratory and clinical data. Clin Exp Immunol. 2010. https://doi.org/10.1111/j.1365-2249.2010.04109.x.

47. Grüber C, Van Stuijvenberg M, Mosca F, Moro G, Chirico G, Braegger CP, et al. Reduced occurrence of early atopic dermatitis because of immunoactive prebiotics among low-atopy-risk infants. J Allergy Clin Immunol. 2010;126:791-7.

48. Moro G, Arslanoglu S, Stahl B, Jelinek J, Wahn U, Boehm G. A mixture of prebiotic oligosaccharides reduces the incidence of atopic dermatitis during the first six months of age. Arch Dis Child. 2006. https://doi. org/10.1136/adc.2006.098251.

49. Difilippo E, Bettonvil M, Willems R, Braber S, Fink-Gremmels J, Jeurink PV, et al. Oligosaccharides in urine, blood, and feces of piglets fed milk 
replacer containing galacto-oligosaccharides. J Agric Food Chem. 2015;63:10862-72.

50. Xu SS, Liu QM, Xiao AF, Maleki SJ, Alcocer M, Gao YY, et al. Eucheuma cottonii sulfated oligosaccharides decrease food allergic responses in animal models by up-regulating regulatory T (Treg) cells. J Agric Food Chem. 2017;65:3212-22.

51. Moussu H, Van Overtvelt L, Horiot S, Tourdot S, Airouche S, Zuercher A et al. Bifidobacterium bifidum NCC 453 promotes tolerance induction in murine models of sublingual immunotherapy. Int Arch Allergy Immunol. 2012;158:35-42.

52. Johansen P, Senti G, Martinez Gomez JM, Storni T, Von Beust BR, Wüthrich $B$, et al. Toll-like receptor ligands as adjuvants in allergenspecific immunotherapy. Clin Exp Allergy. 2005. https://doi.org/10.111 1/j.1365-2222.2005.02384.X.

53. Creticos PS, Schroeder JT, Hamilton RG, Balcer-Whaley SL, Khattignavong $A P$, Lindblad R, et al. Immunotherapy with a ragweed-toll-like receptor 9 agonist vaccine for allergic rhinitis. N Engl J Med. 2006. https://doi. org/10.1542/peds.2007-0846gggg.
54. Drachenberg KJ, Heinzkill M, Urban E, Woroniecki SR. Efficacy and tolerability of short-term specific immunotherapy with pollen allergoids adjuvanted by monophosphoryl lipid A (MPL ${ }^{\circledR}$ ) for children and adolescents. Allergol Immunopathol. 2003. https://doi.org/10.1157/13052432.

55. Rosewich M, Lee D, Zielen S. Pollinex Quattro: an innovative four injections immunotherapy in allergic rhinitis. Hum Vaccin Immunother. 2013. https://doi.org/10.4161/hv.24631.

56. Vázquez-Ortiz M, Álvaro-Lozano M, Alsina L, Garcia-Paba MB, PiquerGibert M, Giner-Muñoz MT, et al. Safety and predictors of adverse events during oral immunotherapy for milk allergy: severity of reaction at oral challenge, specific IgE and prick test. Clin Exp Allergy. 2013;43:92-102.

57. Hofmann AM, Scurlock AM, Jones SM, Palmer KP, Lokhnygina Y, Steele $\mathrm{PH}$, et al. Safety of a peanut oral immunotherapy protocol in children with peanut allergy. J Allergy Clin Immunol. 2009;124:286-91. https://doi. org/10.1016/j.jaci.2009.03.045.
Ready to submit your research? Choose BMC and benefit from:

- fast, convenient online submission

- thorough peer review by experienced researchers in your field

- rapid publication on acceptance

- support for research data, including large and complex data types

- gold Open Access which fosters wider collaboration and increased citations

- maximum visibility for your research: over $100 \mathrm{M}$ website views per year

At BMC, research is always in progress.

Learn more biomedcentral.com/submissions 